\title{
Persistent random walks of charged particles across magnetic field lines
}

\author{
M. Baquero-Ruiz, ${ }^{1}$ A. Fasoli, ${ }^{1}$ I. Furno, ${ }^{1}$ F. Manke, ${ }^{1}$ and P. Ricci ${ }^{1}$ \\ École Polytechnique Fédérale de Lausanne (EPFL), Swiss Plasma Center (SPC), CH-1015 Lausanne, \\ Switzerland
}

(Dated: 29 May 2020)

\begin{abstract}
We investigate the time evolution of the mean location and variance of a charged particle subject to random collisions that are Poisson-distributed. The particle moves on a plane and is subject to a magnetic field applied perpendicular to the plane, so it is constrained to move in circles in the absence of collisions. We develop a procedure that yields analytic expressions of the mean and variance. These results are valid for arbitrary times after the start of the walk, including early on when, on average, less than one collision is expected. As an example of their applicability, we use these expressions to model experimental results and simulations of suprathermal ions propagating in a turbulent plasma in TORPEX.
\end{abstract}

\section{INTRODUCTION}

Transport of charged particles across magnetic field lines is a classic problem in physics. Already in 1915, Townsend ${ }^{1}$ estimated diffusion coefficients of ions in gases. By the 1960s, experiments using magnetized low temperature plasmas had confirmed many theoretical predictions of diffusive behavior, although only under restricted conditions ${ }^{2}$. This prompted a series of investigations, like those of Taylor ${ }^{3}$ and Kurşunoğlu ${ }^{4,5}$. They obtained expressions for the mean square displacement (MSD) of plasma particles across magnetic field lines in the limit of long times, $t \rightarrow \infty$. In 1970, Furuse ${ }^{6}$ found more general expressions valid for all times. Most of these studies used a Langevin ${ }^{7-9}$ approach where the dynamics of the particles is determined by the Lorentz force, a random driving force (usually white noise ${ }^{7,8,10}$ ) and a friction term (usually the Stokes force ${ }^{7}$ ). However, memory kernels that generalize friction can be introduced ${ }^{7,8}$ as well as non-gaussian noise ${ }^{8,9}$. These ideas have given rise to entire fields of research that are still actively pursued.

One such field is fractional Lévy motion ${ }^{11}$, which is used in models of particle transport featuring a non-diffusive character. In the TORoidal Plasma EXperiment ${ }^{12,13}$ (TORPEX), we have used this approach $^{8,14-16}$ to describe transport of suprathermal lithium-6 ions across magnetic field lines in a turbulent plasma. Through appropriate choices of parameters, the Lévy motion models have been shown to reproduce results from simulations and experiments ${ }^{8,15}$ and their evolution over extended periods of time in certain cases $^{14}$, most recently by including truncation effects for particle jumps ${ }^{16}$.

In this work, we study transport without resorting to a random forcing in the form of noise, a friction term, or a memory kernel. Instead, we use a persistent random walk ${ }^{17-19}$ (PRW) approach where particles feature continuous trajectories and finite propagation velocities that lead to correlations in location at short time scales. The random dynamics arises from random instantaneous velocity changes, or collisions, which follow a Poisson distribution with constant rate. This velocity jump pro- cess was studied by Othmer ${ }^{20}$ in the context of biological dispersal studies. Here, however, we include an external force to account for the effect of the magnetic field in the inter-collision motion. We use a method similar to the one developed in Ref. 19 to obtain analytic expressions of particle location statistics, such as the mean and the variance, valid for all times $t \geq 0$ of the random walk.

We consider a $2 \mathrm{D}$ system of a charged particle moving on a plane and a magnetic field applied perpendicular to it. The particle is a random walker undergoing elastic collisions in a 2D PRW. In Sec. II we describe the model and the transport equation that determines the time evolution of the system. In Sec. III, we obtain general expressions for the mean and the variance of the walker location along one axis of motion and then, in Sec. III C, we focus on isotropic initial conditions and symmetric collisions. We compute a complete analytic expression of the variance, valid for all time. Together with results obtained for anisotropic initial conditions (Sec. IIID), we investigate the suitability of the formalism to model suprathermal ion transport in TORPEX (Sec. IV). A summary of the main results, as well as an outlook of future studies, is given in the conclusions, Sec. V.

\section{THEORETICAL FRAMEWORK}

\section{A. Transport equation}

The walker is a particle of charge $q$ and mass $m$ moving in a magnetic field, so it is subject to the Lorentz force. The field $\mathbf{B}$ is applied in the $z$ direction, perpendicular to the plane of motion $x y$, so that the walker dynamics is $2 \mathrm{D}$ (Fig. 1) and can be well described by a location $\mathbf{r}=(x, y)$ and a velocity $\mathbf{v}=\left(v_{x}, v_{y}\right)$. Collisions do not change the magnitude of $\mathbf{v}$ and are assumed to generate instantaneous velocity changes with no z-component.

The existence of collisions makes the walker location random. Let $p(\mathbf{r}, t)$ be the probability density function (PDF) of the random location $\boldsymbol{\rho}(t)=(\chi(t), \psi(t))$ of the particle at time $t$ in the $x y$ plane. Furthermore, let $p(\mathbf{r}, \mathbf{v}, t)$ be the PDF of the walker location and velocity. We note that 


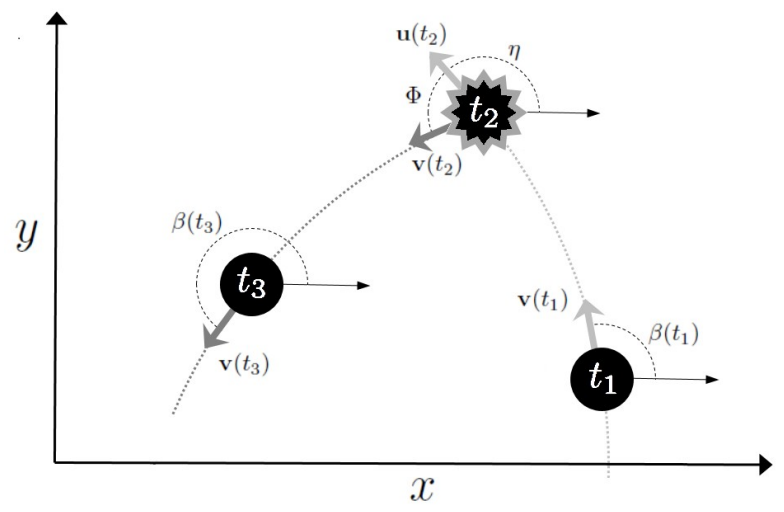

FIG. 1. Depiction of the system dynamics at different times $t_{1}<t_{2}<t_{3}$. A particle with $q<0$ moves in the $x y$ plane subject to $B_{z}>0$. At $t_{1}$ the particle has velocity $\mathbf{v}\left(t_{1}\right)$ and follows a circular trajectory (light gray dotted line). A collision occurs at time $t_{2}$ which instantaneously changes the direction of motion by a random angle $\Phi$. The particle then follows a different circular trajectory (dark gray dotted line) that takes it to a new location at $t_{3}$.

$$
p(\mathbf{r}, t)=\int p(\mathbf{r}, \mathbf{v}, t) d v_{x} d v_{y} \equiv \int p(\mathbf{r}, \mathbf{v}, t) d \mathbf{v} .
$$

For collisions which are distributed Poisson $(t / \tau)$ with constant mean collision time $\tau$, one can generalize the transport equation in Ref. 19 to

$$
\begin{aligned}
\left(\frac{\partial}{\partial t}+\mathbf{v} \cdot \nabla_{\mathbf{r}}+\mathbf{a} \cdot \nabla_{\mathbf{v}}+\gamma\right) & p(\mathbf{r}, \mathbf{v}, t) \\
& =\gamma \int f(\mathbf{u}, \mathbf{v}) p(\mathbf{r}, \mathbf{u}, t) d \mathbf{u}
\end{aligned}
$$

where the symbols $\nabla_{\mathbf{r}}$ and $\nabla_{\mathbf{v}}$ are shorthand for $(\partial / \partial x, \partial / \partial y)$ and $\left(\partial / \partial v_{x}, \partial / \partial v_{y}\right)$, respectively, $\gamma \equiv 1 / \tau$ is the collision rate, and $f(\mathbf{u}, \mathbf{v})$ is a kernel giving the probability that, upon a collision, a walker moving with velocity $\mathbf{u}$ transitions to a velocity v. Similarly as in Ref. 19, $f$ needs to fulfill the normalization condition $\int f(\mathbf{u}, \mathbf{v}) d \mathbf{v}=1$ for all $\mathbf{u}$ to conserve probability. The acceleration $\mathbf{a}$ is given by $\mathbf{a}=(q / m) \mathbf{v} \times \mathbf{B}=\mathbf{v} \times(\Omega \hat{\mathbf{z}})$, where

$$
\Omega=\frac{q}{m} B_{z},
$$

and $\hat{\mathbf{z}}$ is the unitary vector in the direction of $z$ (perpendicular to the $x y$ plane). The values of $q$ and $B_{z}$ can be negative, so Eq. (2) allows in general for negative values of $\Omega$. The quantity $|\Omega| / 2 \pi$ is the cyclotron frequency of the particle.

In all that follows, it is convenient to change the velocity coordinates to the polar representation

$$
\mathbf{v}=\left(v_{x}, v_{y}\right)=v(\cos (\beta), \sin (\beta))
$$

while nevertheless keeping Cartesian coordinates for position (Fig. 1). A straightforward calculation then shows that

$$
\mathbf{a} \cdot \nabla_{\mathbf{v}}=-\Omega \frac{\partial}{\partial \beta}
$$

independent of $v$.

In a collision (Fig. 1), a velocity $\mathbf{u}=u(\cos (\eta), \sin (\eta))$ transitions to $\mathbf{v}$ by changing the original direction $\eta$ by a random angle $\Phi$ such that $\beta=\eta+\Phi$. The angle $\Phi$ is distributed $h(\phi)$, with $\phi \in(-\pi, \pi]$. The PDF $h$ must fulfill certain conditions (see Sec. IIB) but is otherwise arbitrary. The collisions do not alter the speed, so $v=u$. Then

$$
f(\mathbf{u}, \mathbf{v})=\frac{1}{v} \delta(v-u) h(\beta-\eta)=\frac{1}{v} \delta(v-u) h(\phi),
$$

noting that $\int f(\mathbf{u}, \mathbf{v}) d \mathbf{v}=\iint f(\mathbf{u}, \mathbf{v}) v d v d \beta=1$ for all $\mathbf{u}$, as required. With this definition of $f$, the right hand side (RHS) of Eq. (1) becomes

$$
\begin{aligned}
& \int f(\mathbf{u}, \mathbf{v}) p(\mathbf{r}, \mathbf{u}, t) d \mathbf{u} \\
& \quad=\int_{-\pi}^{\pi} h(\phi) p(\mathbf{r}, v \cos (\beta-\phi), v \sin (\beta-\phi), t) d \phi
\end{aligned}
$$

The Lorentz force and the collisions do not change $v$, so $v$ is expected to remain constant throughout the walk. We use the ansatz

$$
p(\mathbf{r}, \mathbf{v}, t)=p(\mathbf{r}, \beta, t) \frac{\delta\left(v-v_{0}\right)}{v}
$$

in Eq. (1), replace the results in Eqs. $(3,4)$, multiply both sides by $v$ and integrate throughout the velocity space to find the transport equation

$$
\begin{gathered}
\left(\frac{\partial}{\partial t}+v_{0} \cos (\beta) \frac{\partial}{\partial x}+v_{0} \sin (\beta) \frac{\partial}{\partial y}-\Omega \frac{\partial}{\partial \beta}+\gamma\right) p(\mathbf{r}, \beta, t) \\
=\gamma \int_{-\pi}^{\pi} h(\phi) p(\mathbf{r}, \beta-\phi, t) d \phi
\end{gathered}
$$

that determines the time evolution of the probability $p(\mathbf{r}, \beta, t)$, which does not depend on $v$. The speed $v_{0}>0$ of the particle is now a constant parameter. We use initial conditions of the form

$$
\left.p(\mathbf{r}, \beta, t)\right|_{t=0}=g(\mathbf{r}) d(\beta),
$$


where $g$ and $d$ are PDFs in 2D position space and angle, respectively. The initial direction of motion, distributed $d$, is therefore assumed to be independent ${ }^{21}$ of the random initial location $\boldsymbol{\rho}_{0}=\left(\chi_{0}, \psi_{0}\right)$, distributed $g$.

\section{B. Transforms}

The function $p(\mathbf{r}, \beta, t)$ is periodic in $\beta$, so it can be expressed $^{22}$ as

$$
\begin{array}{cc}
p(\mathbf{r}, \beta, t) & =\sum_{n=-\infty}^{\infty} p_{n}(\mathbf{r}, t) e^{\imath n \beta} \\
\text { with } \quad & p_{n}(\mathbf{r}, t)=\frac{1}{2 \pi} \int_{-\pi}^{\pi} p(\mathbf{r}, \beta, t) e^{-\imath n \beta} d \beta .
\end{array}
$$

Furthermore, we assume that $p(\mathbf{r}, \beta, t)$ can be Fouriertransformed in $\mathbf{r}$ and Laplace-transformed in $t$, according to

$$
\begin{aligned}
& p(\mathbf{r}, \beta, t) \stackrel{\mathcal{F}}{\rightarrow} p(\boldsymbol{\kappa}, \beta, t) \equiv \int_{-\infty}^{\infty} p(\mathbf{r}, \beta, t) e^{-\imath \boldsymbol{\kappa} \cdot \mathbf{r}} d \mathbf{r}, \\
& p(\mathbf{r}, \beta, t) \stackrel{\mathcal{L}}{\rightarrow} p(\mathbf{r}, \beta, s) \equiv \int_{0}^{\infty} p(\mathbf{r}, \beta, t) e^{-s t} d t
\end{aligned}
$$

where $\boldsymbol{\kappa}=\left(\kappa_{x}, \kappa_{y}\right)$ and $s$ are the conjugate variables of the transforms, so that

$$
p(\mathbf{r}, \beta, t) \stackrel{\mathcal{F}, \mathcal{L}}{\longrightarrow} p(\boldsymbol{\kappa}, \beta, s)=\sum_{n=-\infty}^{\infty} p_{n}(\boldsymbol{\kappa}, s) e^{\imath n \beta}
$$

Using these definitions, we Fourier- and Laplacetransform Eq. (6). We then use Eq. (9) to expand $p(\boldsymbol{\kappa}, \beta, s)$ and obtain (see Appendix A for details)

$$
\begin{aligned}
g(\boldsymbol{\kappa}) d_{n}=( & \left.s-\imath n \Omega+\gamma_{n}\right) p_{n}(\boldsymbol{\kappa}, s) \\
& +\frac{v_{0}}{2}\left(\imath \kappa_{x}+\kappa_{y}\right) p_{n-1}(\boldsymbol{\kappa}, s) \\
& \quad+\frac{v_{0}}{2}\left(\imath \kappa_{x}-\kappa_{y}\right) p_{n+1}(\boldsymbol{\kappa}, s),
\end{aligned}
$$

for all $n \in \mathbb{Z}$, where

$$
\begin{aligned}
& d_{n}=\frac{1}{2 \pi} \int_{-\pi}^{\pi} d(\beta) e^{-\imath n \beta} d \beta, \\
& \gamma_{n}=\gamma\left(1-\int_{-\pi}^{\pi} h(\phi) e^{-\imath n \phi} d \phi\right),
\end{aligned}
$$

assuming that the integrals in the last expressions exist. Evaluation at $\boldsymbol{\kappa}=(0,0) \equiv \mathbf{0}$ leads to

$$
\left.p_{n}(\boldsymbol{\kappa}, s)\right|_{\boldsymbol{\kappa}=\mathbf{0}}=\frac{d_{n}}{s-\imath n \Omega+\gamma_{n}}
$$

since $\left.g(\boldsymbol{\kappa})\right|_{\boldsymbol{\kappa}=\mathbf{0}}=\int g(\mathbf{r}) d \mathbf{r}=1$ (as $g$ is a PDF). Since $d_{0}=1 /(2 \pi)$ and $\gamma_{0}=0$ ( $h$ is also a PDF $)$, evaluation of Eq. (12) at $n=0$ yields $\left.p_{n}(\boldsymbol{\kappa}, s)\right|_{\boldsymbol{\kappa}=\mathbf{0}, n=0}=1 /(2 \pi s)$, which implies $\int p(\mathbf{r}, \mathbf{v}, s) d \mathbf{r} d \mathbf{v}=\int p(\mathbf{r}, \beta, s) d \mathbf{r} d \beta=$ $\left.2 \pi p_{n}(\boldsymbol{\kappa}, s)\right|_{\boldsymbol{\kappa}=\mathbf{0}, n=0}=1 / s$ or, performing the inverse Laplace transform, $\int p(\mathbf{r}, \mathbf{v}, t) d \mathbf{r} d \mathbf{v}=1$. Thus, probability is conserved for all $t \geq 0$.

The transformed function $p_{n}(\boldsymbol{\kappa}, s)$ allows us to compute exact analytic expressions for the moments of $\chi(t)$ without inverse-transforming to the original $(\mathbf{r}, \mathbf{v})$ space $^{19,21}$. For example,

$$
\begin{aligned}
& \left.\frac{\partial}{\partial \kappa_{x}} p_{n}(\boldsymbol{\kappa}, s)\right|_{\substack{\boldsymbol{\kappa}=\mathbf{0} \\
n=0}} \\
& \quad=\left.\frac{\partial}{\partial \kappa_{x}}\left(\frac{1}{2 \pi} \int p(\mathbf{r}, \beta, s) e^{-\imath(\boldsymbol{\kappa} \cdot \mathbf{r}+n \beta)} d \mathbf{r} d \beta\right)\right|_{\substack{\boldsymbol{\kappa}=\mathbf{0} \\
n=0}} \\
& =-\frac{\imath}{2 \pi} \int x p(\mathbf{r}, \beta, s) d \mathbf{r} d \beta \\
& =-\frac{\imath}{2 \pi} \int x p(\mathbf{r}, \beta, s) \frac{\delta\left(v-v_{0}\right)}{v} d \mathbf{r} v d v d \beta \\
& \quad=-\frac{\imath}{2 \pi} \int x p(\mathbf{r}, \mathbf{v}, s) d \mathbf{r} d \mathbf{v}=\mathcal{L}\left\{-\frac{\imath}{2 \pi} \mu_{\chi}(t)\right\}
\end{aligned}
$$

where $\mu_{\chi}(t)$ is the mean value of the walker location along $x$. We can compute the second derivative in a similar way and obtain

$$
\begin{aligned}
& \mu_{\chi}(t)=\mathcal{L}^{-1}\left\{\left.2 \pi \imath \frac{\partial}{\partial \kappa_{x}} p_{n}(\boldsymbol{\kappa}, s)\right|_{\substack{\boldsymbol{\kappa}=\mathbf{0} \\
n=0}}\right\} \\
& \sigma_{\chi}^{2}(t)=\mathcal{L}^{-1}\left\{-\left.2 \pi \frac{\partial^{2}}{\partial \kappa_{x}^{2}} p_{n}(\boldsymbol{\kappa}, s)\right|_{\substack{\boldsymbol{\kappa}=\mathbf{0} \\
n=0}}\right\}-\left[\mu_{\chi}(t)\right]^{2}
\end{aligned}
$$

for the mean and variance of the particle location along $x$. Similar results are found for moments of $\psi(t)$, such as the mean location along $y$ or the variance, by instead performing the derivatives with respect to $\kappa_{y}$. Higher order moments of $\chi(t)$, such as the skewness and kurtosis, can be computed in similar ways.

\section{MEAN AND VARIANCE OF WALKER LOCATION}

\section{A. General expression for mean}

We now differentiate Eq. (10) with respect to $\kappa_{x}$ and evaluate at $\boldsymbol{\kappa}=\mathbf{0}$. Rearranging terms, we obtain

$$
\begin{aligned}
\left.\frac{\partial}{\partial \kappa_{x}} p_{n}(\boldsymbol{\kappa}, s)\right|_{\boldsymbol{\kappa}=\mathbf{0}}=-\frac{\imath \mu_{\chi_{0}} d_{n}}{s-\imath n \Omega+\gamma_{n}} \\
\quad-\frac{\imath v_{0}\left(\left.p_{n-1}(\boldsymbol{\kappa}, s)\right|_{\boldsymbol{\kappa}=\mathbf{0}}+\left.p_{n+1}(\boldsymbol{\kappa}, s)\right|_{\boldsymbol{\kappa}=\mathbf{0}}\right)}{2\left(s-\imath n \Omega+\gamma_{n}\right)}
\end{aligned}
$$

Here, $\mu_{\chi_{0}}=\int x g(\mathbf{r}) d \mathbf{r}=\left\langle\chi_{0}\right\rangle$, the average initial position in $x$. Upon evaluating at $n=0$ and using Eq. (12) to evaluate $\left.p_{n}(\boldsymbol{\kappa}, s)\right|_{\boldsymbol{\kappa}=\mathbf{0}, n= \pm 1}$, we use Eq. (13) to obtain 


$$
\mu_{\chi}(t)-\mu_{\chi_{0}}=\mathcal{L}^{-1}\left\{\frac{v_{0}}{s} \operatorname{Re}\left(\frac{2 \pi d_{1}}{s-\imath \Omega+\gamma_{1}}\right)\right\} .
$$

In this equation, $\operatorname{Re}(\cdot)$ is the real part of the expression in parentheses without considering $s$ as a complex variable. Then, for example, $\operatorname{Re}(s+\imath \Omega)=s$ (since $\Omega$ is real). In general, $d_{1}$ and $\gamma_{1}$ may have a nonzero imaginary part, so the evaluation of Eq. (15) may be nontrivial when $\Omega=0$.

\section{B. General expression for variance}

We differentiate Eq. (10) a second time with respect to $\kappa_{x}$ and evaluate at $\boldsymbol{\kappa}=\mathbf{0}$. Rearranging terms,

$$
\begin{aligned}
\left.\frac{\partial^{2}}{\partial \kappa_{x}^{2}} p_{n}(\boldsymbol{\kappa}, s)\right|_{\boldsymbol{\kappa}=\mathbf{0}}=-\frac{\left(\sigma_{\chi_{0}}^{2}+\mu_{\chi_{0}}^{2}\right) d_{n}}{s-\imath n \Omega+\gamma_{n}} \\
-\frac{\imath v_{0}\left(\left.\frac{\partial}{\partial \kappa_{x}} p_{n-1}(\boldsymbol{\kappa}, s)\right|_{\boldsymbol{\kappa}=\mathbf{0}}+\left.\frac{\partial}{\partial \kappa_{x}} p_{n+1}(\boldsymbol{\kappa}, s)\right|_{\boldsymbol{\kappa}=\mathbf{0}}\right)}{s-\imath n \Omega+\gamma_{n}}
\end{aligned}
$$

where $\sigma_{\chi_{0}}^{2}$ is the variance of $\chi_{0}$, the initial location in $x$. We then evaluate this expression at $n=0$, using Eqs. (12, 14) to find the value of the derivatives on the RHS. Finally, we use Eq. (13) to obtain the general expression for the variance of the walker

$$
\begin{aligned}
& \sigma_{\chi}^{2}(t)-\sigma_{\chi_{0}}^{2}+\left[\mu_{\chi}(t)-\mu_{\chi_{0}}\right]^{2} \\
& =\mathcal{L}^{-1}\left\{\frac{v_{0}^{2}}{s^{2}} \operatorname{Re}\left[\frac{1}{s-\imath \Omega+\gamma_{1}}\left(1+\frac{2 \pi d_{2} s}{s-\imath 2 \Omega+\gamma_{2}}\right)\right]\right\} .
\end{aligned}
$$

The function $\operatorname{Re}(\cdot)$ follows the same convention as in Eq. (15).

As a straightforward example of the applicability of Eq. (16), we consider a case with no $B$-field but $\gamma \neq 0$. Every time the particle undergoes a collision, it reverses its direction of motion, $h(\phi)=\delta(\phi-\pi)$. The initial location is $x=0$ with complete certainty, so $g(\mathbf{r})=\delta(\mathbf{r})$, and the initial direction of motion is parallel to the $x$-axis and with equal probability of going either side, $d(\beta)=(1 / 2)(\delta(\beta)+\delta(\beta-\pi))$. Using the definitions in Sec. IIB, one can establish the values of the different parameters appearing in Eqs. $(15,16)$, such as $\Omega=0$, $d_{1}=0, d_{2}=1 /(2 \pi), \gamma_{1}=2 \gamma, \gamma_{2}=0, \mu_{\chi_{0}}=0$ and $\sigma_{\chi_{0}}^{2}=0$. Equation (15) then straightforwardly leads to $\mu_{\chi}(t)=0$ for all $t \geq 0$. Equation (16) leads to

$$
\begin{aligned}
\sigma_{\chi}^{2}(t)= & \mathcal{L}^{-1}\left\{\frac{v_{0}^{2}}{s^{2}} \operatorname{Re}\left(\frac{2}{s+2 \gamma}\right)\right\} \\
& =\frac{v_{0}^{2}}{2 \gamma^{2}}\left[(2 \gamma t-1)+e^{-2 \gamma t}\right]
\end{aligned}
$$

a result consistent with expected results in $1 \mathrm{D} \mathrm{PRW} \mathrm{PW}^{19,20}$.

\section{Isotropic initial conditions and symmetric collisions}

If any direction of motion on the $x y$ plane is equally probable at $t=0$, the initial motion is isotropic. In that case,

$$
d(\beta)=\frac{1}{2 \pi} \quad \text { for all } \beta \in(-\pi, \pi]
$$

A direct computation shows that this choice of $d$ yields $d_{n}=0$ for all $n \neq 0$. Equation (15) then shows that $\mu_{\chi}(t)=\mu_{\chi_{0}}$ for all $t \geq 0$, independent of the $B$-field, collisions, and $h$.

If $h(\phi)=h(-\phi)$ for all $\phi \in(-\pi, \pi)$, collisions are symmetric, having equal probabilities of deviating the trajectory to either side with respect to the pre-collision direction of motion. In that situation, Eq.(11) leads to $\operatorname{Im}\left(\gamma_{1}\right)=0$. For example, if

$$
h(\phi)= \begin{cases}\frac{1}{2 \phi_{M}} & \text { if } \phi \in\left(-\phi_{M}, \phi_{M}\right) \\ 0 & \text { otherwise }\end{cases}
$$

where $\phi_{M} \in(0, \pi)$ is a constant parameter, then

$$
\begin{aligned}
\gamma_{1} & =\gamma\left(1-\frac{\sin \left(\phi_{M}\right)}{\phi_{M}}\right) \\
& \approx \frac{\gamma \phi_{M}^{2}}{6} \quad\left(\text { if } \phi_{M}<1\right)
\end{aligned}
$$

The last line is an approximation when scattering angles are small. In that case, there can be an interpretation of $\gamma_{1}$ as an angle change (squared), due to collisions, per unit time. A larger value of $\gamma_{1}$ can be obtained by either an increase in $\gamma$ or larger $\sigma_{M}$. On the contrary, if $\phi_{M} \rightarrow \pi$ (isotropic post-collision direction PDF) then $\gamma_{1} \rightarrow \gamma$.

Assuming isotropic initial conditions and symmetric collisions, Eqs. $(15,16)$ yield

$$
\begin{aligned}
\sigma_{\chi}^{2}(t) & -\sigma_{\chi_{0}}^{2}=\mathcal{L}^{-1}\left\{\frac{v_{0}^{2}}{s^{2}} \operatorname{Re}\left(\frac{1}{s+\gamma_{1}-\imath \Omega}\right)\right\} \\
= & \frac{v_{0}^{2} \gamma_{1}}{\gamma_{1}^{2}+\Omega^{2}} t+v_{0}^{2} \frac{\Omega^{2}-\gamma_{1}^{2}}{\left(\gamma_{1}^{2}+\Omega^{2}\right)^{2}} \\
& +\frac{v_{0}^{2} e^{-\gamma_{1} t}}{\left(\gamma_{1}^{2}+\Omega^{2}\right)^{2}}\left[\left(\gamma_{1}^{2}-\Omega^{2}\right) \cos (\Omega t)-2 \Omega \gamma_{1} \sin (\Omega t)\right]
\end{aligned}
$$

This expression is independent of the sign of $\Omega$.

In the limit $\gamma_{1} t \gg 1$, Eq. (20) leads to diffusive behavior characterized by $\sigma_{\chi}^{2} \sim t^{\lambda}$ with $\lambda=1$. Indeed, $\sigma_{\chi}^{2}(t) / t \approx\left(v_{0}^{2} \gamma_{1}\right) /\left(\gamma_{1}^{2}+\Omega^{2}\right)$, as expected from the known result by Taylor $^{3}$. At early times of the walk, when $t \ll|\Omega|^{-1}$ and $t \ll \gamma_{1}^{-1}, \sigma_{\chi}^{2}(t) \approx \sigma_{\chi_{0}}^{2}+\left(v_{0}^{2} / 2\right) t^{2}$. Provided $\sigma_{\chi_{0}} \ll v_{0} /|\Omega|$, the motion will instead be ballistic, with $\lambda=2$. 


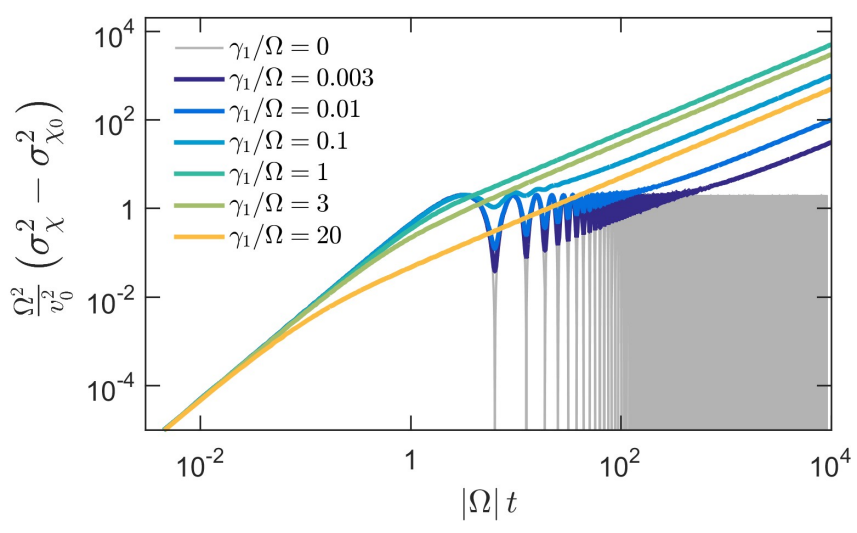

FIG. 2. Evolution of $\sigma_{\chi}^{2}(t)$ for different values of $\gamma_{1} / \Omega$.

In the case of no collisions $(\gamma=0)$, Eq. (20) shows that $\sigma_{\chi}^{2}(t)=\sigma_{\chi_{0}}^{2}+\left(v_{0} / \Omega\right)^{2}(1-\cos (\Omega t))$, a non-decaying oscillatory motion with angular frequency $\Omega$. If, instead, we take $B_{z}=0$ but $\gamma_{1} \neq 0$, then we obtain $\sigma_{\chi}^{2}(t)=$ $\sigma_{\chi_{0}}^{2}+\left(v_{0} / \gamma_{1}\right)^{2}\left[\left(\gamma_{1} t-1\right)+e^{-\gamma_{1} t}\right]$, similar to 1D PRW (see Sec. IIIB) but at a slightly slower pace (notice the different factor preceding the square brackets). The case $\gamma=0, B_{z}=0$, can be computed as a limiting situation of Eq.(20), but is easier to obtain from Eq. (16). It yields $\sigma_{\chi}^{2}(t)=\sigma_{\chi_{0}}^{2}+\left(v_{0}^{2} / 2\right) t^{2}$, identical in value to early stages of the walk at arbitrary $\gamma_{1}$ and $B_{z}$ (as seen before), but valid for all time $t \geq 0$.

If $B_{z} \neq 0$, Eq. (20) can be used to express $\left(\Omega^{2} / v_{0}^{2}\right)\left(\sigma_{\chi}^{2}(t)-\sigma_{\chi_{0}}^{2}\right)$ in terms only of $\Omega t$ and $\gamma_{1} / \Omega$. This means that the functional form of the time evolution only depends on the ratio of $\gamma_{1}$ to $\Omega$ and not on the individual values. Figure 2 shows the evolution of the variance for different $\gamma_{1} / \Omega$. Whenever $\gamma_{1} /|\Omega| \lesssim 1$, oscillations occur up to $|\Omega| t \approx 3|\Omega| / \gamma_{1}$ with no significant increment of the amplitude. Then, the variance transitions to having a linear dependency with time ${ }^{19}$, recovering the diffusive behavior expected from the preceding discussion. When $\gamma_{1} /|\Omega| \gtrsim 1$, no oscillations are visible and an initial slope of value 2 (ballistic) in the plot transitions smoothly to 1 (diffusive).

Interestingly, given a particular choice of $\gamma_{1} / \Omega$, the long term value of $\left(\Omega^{2} / v_{0}^{2}\right) \sigma_{\chi}^{2}(t)$ is approximately equal to the one obtained using the reciprocal, $\left(\gamma_{1} / \Omega\right)^{-1}$. This is because

$$
\begin{aligned}
& \left(\frac{\Omega^{2}}{v_{0}^{2}}\right) \sigma_{\chi}^{2}(t) \stackrel{\stackrel{\gamma_{1} t \gg 1}{\longrightarrow}}{\Omega^{2} \gamma_{1}} \frac{\left(\gamma_{1} / \Omega\right)}{\gamma_{1}^{2}+\Omega^{2}} t=\frac{\left(\Omega / \gamma_{1}\right)}{1+\left(\gamma_{1} / \Omega\right)^{2}}(\Omega t)=\frac{(\Omega t) .}{\left(\Omega / \gamma_{1}\right)^{2}+1} .
\end{aligned}
$$

Then, whenever $\gamma_{1}=|\Omega|$, one obtains a maximum variance $^{3}$ for given $\Omega$ and $v_{0}$. This result can be verified in Fig. 2 in the region where $|\Omega| t \gtrsim 10$.

All these results are in good agreement with numerical simulations performed in MATLAB (see Appendix B).

\section{Anisotropic initial conditions and isotropic collisions}

Collisions become isotropic by letting $\phi_{M} \rightarrow \pi$ in Eq. (18). Equation (11) then shows that $\gamma_{n}=\gamma$ for all $n \neq 0$.

We now consider anisotropic initial conditions generated with a von Mises $^{23}$ distribution, defined as

$$
d\left(\beta ; \beta_{0}, c\right)=\frac{e^{c \cos \left(\beta-\beta_{0}\right)}}{2 \pi I_{0}(c)},
$$

with mean angle $\beta_{0}$ and width set by the parameter $c \geq$ 0. $I_{n}$ is the modified Bessel function of the first kind ${ }^{22}$ of order $n \geq 0$. If $c=0$ then we recover an isotropic distribution (Eq. (17)). If $c \gg 1$, then $d\left(\beta ; \beta_{0}, c\right)$ is approximately Normal with mean $\beta_{0}$ and variance $1 / c$. From the characteristic function ${ }^{23}$ it is straightforward to show that

$$
d_{n}=\frac{I_{n}(c)}{2 \pi I_{0}(c)} e^{-i n \beta_{0}},
$$

valid for $n \geq 0$.

Replacing $\gamma_{1}$ and $d_{1}$ in Eq. (15) leads to a complicated analytic expression for the mean of $\chi$ for arbitrary values of $\beta_{0}$. Evaluation at $\beta_{0}=0$ yields an example with the simpler expression

$$
\begin{aligned}
& \mu_{\chi}(t)-\mu_{\chi_{0}}= \\
& \quad \frac{I_{1}(c)}{I_{0}(c)} \frac{v_{0}}{\gamma^{2}+\Omega^{2}}\left[\Omega e^{-\gamma t} \sin (\Omega t)+\gamma\left(1-e^{-\gamma t} \cos (\Omega t)\right)\right] .
\end{aligned}
$$

The case $c=0$ recovers $\mu_{\chi}(t)=\mu_{\chi_{0}}$ as expected from Sec. III C.

The situation is still more complicated for the variance. Replacement of $\gamma_{1}, \gamma_{2}, d_{2}$ and $\mu_{\chi}(t)$ in Eq. (16) yields analytic results that are cumbersome to present explicitly. The results are nevertheless plotted in Fig. 3 for $\beta_{0}=0, \pi / 2$, where the biases in initial direction are seen to have an impact on the evolution of $\sigma_{\chi}^{2}$. Interestingly, transport can behave supraballistically, with $\sigma_{\chi}^{2} \sim t^{3}$, in certain situations. The reason for this is likely a diffusion of velocities along $x$ caused be early collisions, as explained in Ref. 19. The effect is more clearly seen at large values of $c$ since, for those cases, the distribution of initial velocities has smaller width.

\section{APPLICATION OF MODEL TO SIMULATIONS OF SUPRATHERMAL IONS IN TORPEX}

\section{A. Suprathermal ion studies in TORPEX}

As an example of application of the results of Sec. III, we consider the case of suprathermal ion transport in TORPEX (Fig. 4). 


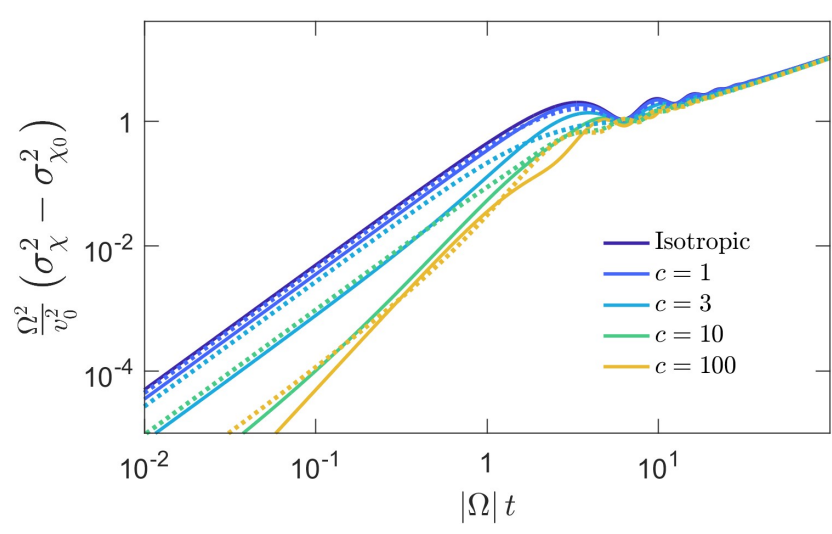

FIG. 3. Evolution of $\sigma_{\chi}^{2}(t)$ for $\gamma / \Omega=0.1$ and different values of the width parameter $c$. Solid lines correspond to $\beta_{0}=0$ and dotted lines to $\beta_{0}=\pi / 2$ (same color coding). The case $c=0$ yields an isotropic result compatible with the model of Sec. III C. Notice the large slope at $|\Omega t| \lesssim 1$ for the curves with $c \gtrsim 10$.

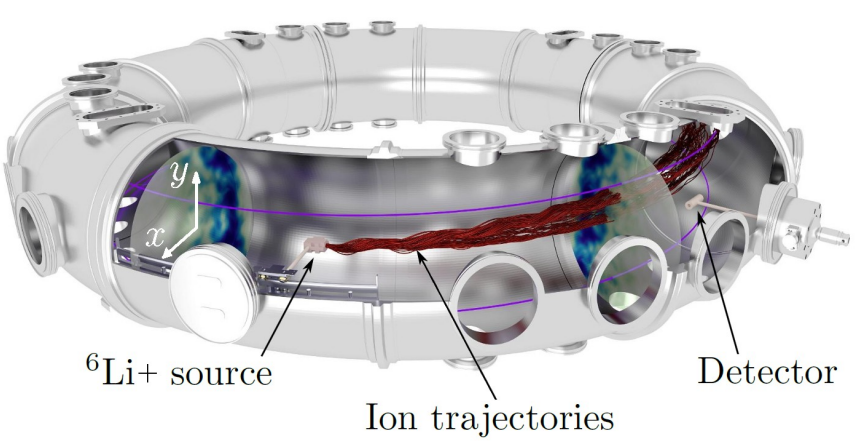

FIG. 4. Suprathermal ions in TORPEX. We use a magnetic configuration with open helical lines (depicted with the solid purple line). Lithium-6 ions are injected in a turbulent hydrogen plasma using a miniature source. As they propagate, the ions spread in the perpendicular direction ( $x y$ plane). A detector measures the ions arriving at different $(x, y)$ locations. The toroidal distance between source and detector can be changed to resolve different propagation times.

TORPEX ${ }^{12,13}$ is a basic toroidal plasma physics device located at the Swiss Plasma Center in Lausanne, Switzerland, of major radius $1 \mathrm{~m}$ and minor radius $20 \mathrm{~cm}$. In the typical Simple Magnetized Torus (SMT) configuration, where a small vertical field $B_{v} \approx 2 \mathrm{mT}$ is superposed on a dominant toroidal field $B_{\phi} \approx 74 \mathrm{mT}$, hydrogen plasmas of densities $n_{e}=10^{15}-10^{17} \mathrm{~m}^{-3}$, electron temperatures $T_{e} \approx 1-5 \mathrm{eV}$ and plasma potentials $V_{p}=10-20 \mathrm{~V}$, are produced which exhibit turbulent behavior ${ }^{24}$. Plasma structures, elongated along the $B$-field, can detach from the main plasma and propagate radially outward ${ }^{13,25,26}$, giving rise to so called "blobs".

In these conditions, a beam of lithium- 6 ions $\left({ }^{6} \mathrm{Li}+\right)$ is injected with an energy much larger than $T_{e}$ (see Fig. 4).
These suprathermal ions have no effect on the plasma or the fields (they act as tracers). They propagate mostly parallel to the helical $B$-field lines, but the existence of a non-zero curvature and $\nabla B$ leads them to drift across field lines. Furthermore, the ions interact with the electric field produced by the fluctuating $V_{p}$. Plasma structures are $B$-field aligned ${ }^{13}$, so $V_{p}$ has negligible gradients parallel to the field. The ${ }^{6} \mathrm{Li}+$ are then subject to a fluctuating force that affects their perpendicular motion and leads to 2D transport on the $x y$ plane (Fig. 4).

Extensive studies ${ }^{8,13,15}$ of this setup have been performed experimentally and with simulations. The simulations use the GBS $\operatorname{code}^{27}$ to generate the turbulent electric field where particles are traced. The results show non-diffusive perpendicular transport features which change with ${ }^{6} \mathrm{Li}+$ propagation time and with ion injection energy.

\section{B. Simulations and PRW model}

The PRW formalism developed in Secs. II, III can be used to model the perpendicular motion of the ${ }^{6} \mathrm{Li}+$ by replacing the effect of the fluctuating potential with random changes in direction on $x y$. Since the model assumes a constant $B$-field perpendicular to $x y$, we look only at location statistics along $x$ to avoid complications arising from the drifts (directed along $y$, see Fig. 4) caused by the $3 \mathrm{D}$ spatial variation of $\mathbf{B}$. Furthermore, the model assumes no changes in ion kinetic energy, even though some heating may occur due to large electric field gradients ${ }^{28}$. $3 \mathrm{D}$ effects arising from plasma variations along $B$-field lines are also neglected, since they are expected not to be important (see Sec. IV A). The formalism is admittedly a simplification, but it does capture some of the main features of experiments and GBS simulation results, as described next.

We consider pre-existing and well analyzed experimental data and simulations ${ }^{8,13,15}$ of suprathermal ions with injection energies $\approx 30 \mathrm{eV}$ and $\approx 70 \mathrm{eV}$. In both cases, the injection is performed at an angle of $5.7^{\circ} \pm 4.6^{\circ}$ with respect to the $B$-field lines. The angle spread, as well as variations in the initial energy, give rise to a spread in initial perpendicular velocities.

Figure 5 shows experimental data of measured variance against $|\Omega| t$. The value of $\sigma_{\chi}^{2}$ and the error bars are computed as the square of the measurements of ${ }^{6} \mathrm{Li}+$ ion beam width versus toroidal propagation distance (i.e., separation between source and detector) in Refs. 8 and 15. The initial location spreading $\sigma_{\chi_{0}}^{2} \approx(0.3 \mathrm{~cm})^{2}=$ $0.09 \mathrm{~cm}^{2}$ is determined from the same observations. The mapping to $|\Omega| t$ is performed using $\Omega / 2 \pi=188 \mathrm{kHz}^{8,15}$ and ${ }^{6} \mathrm{Li}+$ velocities $4.70 \times 10^{4} \mathrm{~m} / \mathrm{s}$ and $3.08 \times 10^{4} \mathrm{~m} / \mathrm{s}$ in the direction parallel to $\mathbf{B}$, for $70 \mathrm{eV}$ and $30 \mathrm{eV}$, respectively. There is a small uncertainty in the average value of $|\Omega| t$ of the ions detected at a given detector distance, due mainly to the spread in experimental injection energies $^{8}$, which is estimated to be $<5 \%$. Figure 5 also 

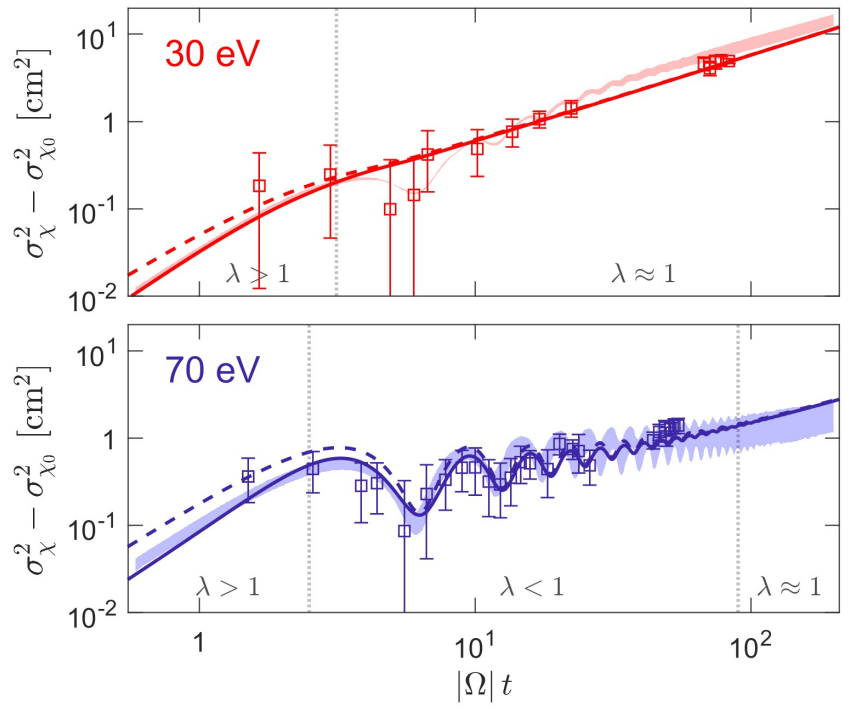

FIG. 5. PRW model (lines), experimental data (squares) and GBS simulations (bands) of ${ }^{6} \mathrm{Li}+$ location variance (along $x$ ) for $30 \mathrm{eV}$ (top) and $70 \mathrm{eV}$ (bottom). Data error bars are the $1 \sigma$ uncertainties corresponding to the square of the $1 \sigma$ errors in Refs. 8 and 15. Dashed lines correspond to isotropic initial conditions and solid lines to injection biased in the $+x$ direction. See text for a discussion on parameter values. Initially, transport is ballistic with $\lambda \approx 2$, while at long times $(|\Omega| t \gtrsim 100)$ it is diffusive $(\lambda \approx 1)$. A subdiffusive $(\lambda<1)$ regime is observed in the $70 \mathrm{eV}$ case in the region between vertical dotted lines.

shows the GBS-simulated evolution of the spreading of ${ }^{6} \mathrm{Li}+$ along $x$ for the two injection energies. The shaded bands represent ranges of simulation results consistent with experimental uncertainties.

To model the $30 \mathrm{eV}$ case (see Fig. 5), we choose $\gamma_{1} / \Omega=0.6$ (see Sec. III), as it exhibits a rapid transition between ballistic $\left(\sigma_{\chi}^{2} \sim t^{2}\right)$ and diffusive $\left(\sigma_{\chi}^{2} \sim t\right)$ regimes. With isotropic initial conditions (Sec. IIIC), a value $v_{0}^{2} / \Omega^{2}=0.13 \mathrm{~cm}^{2}$ yields good agreement with experiments. Compared to the simulations there is, however, an initial overestimation of the variance that can be reduced by assuming an injection biased in the $+x$ direction $(c=1.8, \gamma / \Omega=0.6$, Sec. IIID). This choice cannot reproduce the simulated variance oscillations at times $3 \lesssim|\Omega| t \lesssim 30$. Actually, no choice of parameters was found that could accomplish this, suggesting that the simplicity of the PRW model may make it unsuitable to capture this effect in the $30 \mathrm{eV}$ case.

To model the $70 \mathrm{eV}$ simulations, we choose $v_{0}^{2} / \Omega^{2}=$ $0.39 \mathrm{~cm}^{2}$ consistent with the fact that these simulations include energies up to $\approx 75 \mathrm{eV}$, a factor of 3 larger than the $\approx 25 \mathrm{eV}$ that can be found in the $30 \mathrm{eV}$ case. We also choose $\gamma_{1} / \Omega=0.03$ as it allows for oscillations of $\sigma_{\chi}^{2}$ until $|\Omega| t \approx 100$. The biased injection (with $c=1.8, \gamma / \Omega=0.016$ ) yields a better fit to the simulations than the isotropic one (Fig. 5), especially at early times $|\Omega| t \lesssim 1$, but both the isotropic and biased cases capture the main features of the experimental data, with a superdiffusive character at early times that becomes subdiffusive and, afterwards, progressively transitions to diffusive.

A physical interpretation of these results can be made by focusing on isotropic injection (Sec. III C). According to Eqs. $(11,18,19)$, the value of $\gamma_{1} / \Omega$ can be regarded, approximately, as an average cumulative deflection angle per gyro-cycle, provided the deflection angle per collision is small $(\ll 1)$. Then, the results above suggest that the effect of the turbulent electric field on the transport of ${ }^{6} \mathrm{Li}+$ ions may be similar to a collection of random, small angle, changes in direction with fixed total expected deflection per gyro-cycle. The higher value of $\gamma_{1} / \Omega$ of the $30 \mathrm{eV}$ case (see Fig. 5) suggests a larger cumulative deflection compared to $70 \mathrm{eV}$, an observation possibly consistent with gyro-averaging ${ }^{8,15}$ of $V_{p}$ structures.

\section{CONCLUSIONS}

We have developed a 2D persistent random walk model of a charged particle in a magnetic field. The particle is subject to elastic collisions which are distributed Poisson with constant rate.

Using a procedure similar to the one in Ref. 19, we obtain general expressions for the mean and variance of the particle location. The formalism developed in Secs. IIIII can be straightforwardly used to compute higher order moments if desired. Under the assumption of isotropic initial conditions and symmetric collisions (Sec. III C), we compute an exact analytical expression of the variance (along the $x$ axis), valid for all time $t \geq 0$, that has been tested against numerical simulations (Appendix B). We also investigate anisotropic initial conditions (with isotropic collisions, Sec. IIID) and show that differences in initial conditions can have an impact on the properties of transport.

The results are used to model the time evolution of the variance in experiments and simulations of suprathermal ions in TORPEX (Sec. IV B). The model is seen to capture the main features of transport across magnetic field lines as well as the evolution of the character of transport over time. While other models based on Lévy motion $^{14,16}$ can describe asymmetrical and non-diffusive behavior in a single regime at all times, the presented PRW model is successfully able to incorporate a variety of transitions between different transport regimes, while always converging to diffusion in the long term limit. The PRW model therefore offers a consistent method to describe transient non-diffusive transport, without the requirement of invoking non-markovian or non-local assumptions. The PRW and Lévy motion approaches can therefore complement each other, depending on the specific system and time frames under investigation.

The model has been developed in the context of plasma physics and may find further applications in this field, for 
example in studies of particle confinement in tokamaks. It may also be relevant in studies of any $2 \mathrm{D}$ system where particles undergo random elastic collisions and in biology, for example in studies of cell motion ${ }^{29}$.

\section{ACKNOWLEDGMENTS}

This work has been carried out within the framework of the EUROfusion Consortium and has received funding from the Euratom research and training programme 2014 - 2018 and 2019 - 2020 under grant agreement No 633053. The views and opinions expressed herein do not necessarily reflect those of the European Commission.

This work was supported in part by the Swiss National Science Foundation.

\section{Appendix A: Transforming the transport equation}

The Fourier and Laplace transform of Eq. (6) yields

$$
\begin{gathered}
\left(s+\imath \kappa_{x} v_{0} \cos (\beta)+\imath \kappa_{y} v_{0} \sin (\beta)-\Omega \frac{\partial}{\partial \beta}+\gamma\right) p(\boldsymbol{\kappa}, \beta, s) \\
=g(\boldsymbol{\kappa}) d(\beta)+\gamma \int_{-\pi}^{\pi} h(\phi) p(\boldsymbol{\kappa}, \beta-\phi, s) d \phi
\end{gathered}
$$

The first term on the RHS corresponds to the initial conditions appearing from the transform of $(\partial / \partial t) p(\mathbf{r}, \beta, t)$.

The function $p(\boldsymbol{\kappa}, \beta, s)$ can be expressed as a Fourier series (Eq. (9)). Replacing it in Eq. (A1) yields, first,

$$
\begin{aligned}
& {\left[\imath \kappa_{x} v_{0} \cos (\beta)+\imath \kappa_{y} v_{0} \sin (\beta)\right] p(\boldsymbol{\kappa}, \beta, s)} \\
& =\sum_{n=-\infty}^{\infty} p_{n}(\boldsymbol{\kappa}, s)\left[\imath \kappa_{x} v_{0} \cos (\beta)+\imath \kappa_{y} v_{0} \sin (\beta)\right] e^{\imath n \beta} \\
& =\frac{v_{0}}{2} \sum_{n=-\infty}^{\infty} p_{n}(\boldsymbol{\kappa}, s)\left(\imath \kappa_{x}+\kappa_{y}\right) e^{\imath(n+1) \beta} \\
& \quad+\frac{v_{0}}{2} \sum_{n=-\infty}^{\infty} p_{n}(\boldsymbol{\kappa}, s)\left(\imath \kappa_{x}-\kappa_{y}\right) e^{\imath(n-1) \beta} \\
& =\frac{v_{0}}{2} \sum_{n=-\infty}^{\infty} p_{n-1}(\boldsymbol{\kappa}, s)\left(\imath \kappa_{x}+\kappa_{y}\right) e^{\imath n \beta} \\
& \quad+\frac{v_{0}}{2} \sum_{n=-\infty}^{\infty} p_{n+1}(\boldsymbol{\kappa}, s)\left(\imath \kappa_{x}-\kappa_{y}\right) e^{\imath n \beta}
\end{aligned}
$$

The third line follows from the basic identities $\cos (\beta)=$ $(1 / 2)\left(e^{\imath \beta}+e^{-\imath \beta}\right)$ and $\sin (\beta)=(1 / 2 \imath)\left(e^{\imath \beta}-e^{-\imath \beta}\right)$. Next,

$$
\begin{aligned}
\Omega & \frac{\partial}{\partial \beta} p(\boldsymbol{\kappa}, \beta, s) \\
& =\sum_{n=-\infty}^{\infty} p_{n}(\boldsymbol{\kappa}, s)\left(\Omega \frac{\partial}{\partial \beta}\right) e^{\imath n \beta} \\
& =\sum_{n=-\infty}^{\infty} p_{n}(\boldsymbol{\kappa}, s)(\imath n \Omega) e^{\imath n \beta}
\end{aligned}
$$

where we have assumed that it is possible to differentiate the series term by term. A similar assumption is made for the second term on the RHS of Eq. (A1), this time regarding the integrals. We have

$$
\begin{aligned}
& \gamma \int_{-\pi}^{\pi} h(\phi) p(\boldsymbol{\kappa}, \beta-\phi, s) d \phi \\
& =\gamma \int_{-\pi}^{\pi} h(\phi)\left[\sum_{n=-\infty}^{\infty} p_{n}(\boldsymbol{\kappa}, s) e^{\imath n(\beta-\phi)}\right] d \phi \\
& =\sum_{n=-\infty}^{\infty} p_{n}(\boldsymbol{\kappa}, s)\left(\gamma \int_{-\pi}^{\pi} h(\phi) e^{-\imath n \phi} d \phi\right) e^{\imath n \beta}
\end{aligned}
$$

which, together with Eqs. (A2, A3) and using the expansion of $d(\beta)$ in a Fourier series (Eq. (11)), leads to the complete expanded form of Eq. (A1)

$$
0=\sum_{n=-\infty}^{\infty}\left[\left(s-\imath n \Omega+\gamma_{n}\right) p_{n}(\boldsymbol{\kappa}, s)+\frac{v_{0}}{2}\left(\imath \kappa_{x}+\kappa_{y}\right) p_{n-1}(\boldsymbol{\kappa}, s)+\frac{v_{0}}{2}\left(\imath \kappa_{x}-\kappa_{y}\right) p_{n+1}(\boldsymbol{\kappa}, s)-g(\boldsymbol{\kappa}) d_{n}\right] e^{\imath n \beta}
$$


Here $\gamma_{n}$ is defined as in Eq. (11). This series can only yield zero if all the coefficients accompanying the exponentials are zero, so the term in brackets is zero for all $n$. This result is equivalent to Eq. (10).

\section{Appendix B: Numerical tests}

We test the main results of Sec. IIIC by comparing them with numerical estimates obtained with a MATLAB code ${ }^{19}$. The code generates $N=10^{5}$ initial conditions distributed $g$ and $d$, and uses them to integrate $N$ random trajectories with discrete time steps $\Delta t=$ $\left(10^{3} \max (\gamma,|\Omega|)\right)^{-1}$. For each trajectory $\mathrm{n}=1,2, \ldots, N$, the location $\left(x_{\mathrm{n}}(t), y_{\mathrm{n}}(t)\right)$ and the direction of motion $\beta_{\mathrm{n}}(t)$ is updated according to

$$
\begin{aligned}
& x_{\mathrm{n}}(t+\Delta t)=x_{\mathrm{n}}(t)+v_{0} \Delta t \cos \left(\beta_{\mathrm{n}}(t)\right) \\
& y_{\mathrm{n}}(t+\Delta t)=y_{\mathrm{n}}(t)+v_{0} \Delta t \sin \left(\beta_{\mathrm{n}}(t)\right) \\
& \beta_{\mathrm{n}}(t+\Delta t)= \begin{cases}\beta_{\mathrm{n}}(t)-\Omega \Delta t & \text { if no collision happens } \\
\beta_{\mathrm{n}}(t)+\Phi_{\mathrm{n}} & \text { if there is a collision. }\end{cases}
\end{aligned}
$$

The random angle $\Phi_{\mathrm{n}}$ is generated with a symmetric PDF $h$ as defined in Eq. (18). The motion is initially isotropic, with $d$ as in Eq. (17). We choose $g$ to be 2D Normal ${ }^{21}$ with mean $\left\langle\boldsymbol{\rho}_{0}\right\rangle=\mathbf{0}$, zero $x$-y correlation (circular shape) and $\sigma_{\chi_{0}}^{2}=\sigma_{\psi_{0}}^{2}=\left(10^{-4} v_{0} / \Omega\right)^{2}$. The estimation of the mean and variance at time $t$ is computed using the appropriate sample statistics of the $N$ locations at $t$. The implementation of Poisson collisions, the choice of $\Delta t$ and more details of the code are described in Ref. 19 .

Figure 6 shows a comparison of simulation results (markers) to predictions based on the theory of Sec. III C (solid lines). The purple $\mathrm{x}$-markers correspond to the simulation parameters $v_{0}=10 \mathrm{~m} / \mathrm{s}, \Omega=2 \pi \mathrm{rad} / \mathrm{s}, \gamma=$ $31.42 \mathrm{~s}^{-1}$ and $\phi_{M}=\pi$, which lead to $\gamma_{1} / \Omega=5$; blue triangles to $v_{0}=5 \mathrm{~m} / \mathrm{s}, \Omega=-5 \pi \mathrm{rad} / \mathrm{s}, \gamma=22.44 \mathrm{~s}^{-1}$ and $\phi_{M}=3 \pi / 4$ (for $\gamma_{1} / \Omega=-1$ ); blue-green crosses to $v_{0}=2 \mathrm{~m} / \mathrm{s}, \Omega=8 \pi \mathrm{rad} / \mathrm{s}, \gamma=6.91 \mathrm{~s}^{-1}$ and $\phi_{M}=\pi / 2$ (for $\gamma_{1} / \Omega=0.1$ ); and yellow circles to $v_{0}=1 \mathrm{~m} / \mathrm{s}$, $\Omega=-11 \pi \mathrm{rad} / \mathrm{s}, \gamma=0.35 \mathrm{~s}^{-1}$ and $\phi_{M}=\pi\left(\right.$ for $\gamma_{1} / \Omega=$ $-0.01)$. These cases, which reproduce situations with differing transport features, show very good agreement with the theoretical curves of the mean and the variance along $x$.

${ }^{1}$ J.S. Townsend, Electricity in gases, Oxford University Press, Cambridge, England, 1915, pp. 96-100.

${ }^{2}$ F.C. Hoh, Low-temperature plasma diffusion in a magnetic field, Rev. Mod. Phys. 34, 267 (1962).

${ }^{3}$ J.B. Taylor, Diffusion of plasma across a magnetic field, Phys. Rev. Lett. 6, 262 (1961).

${ }^{4}$ B. Kurşunoğlu, Brownian motion in a magnetic field, Ann. Phys. 17, 259 (1962).

${ }^{5}$ B. Kurşunoğlu, Brownian motion in a magnetic field, Phys. Rev. 132, 21 (1963).

${ }^{6} \mathrm{H}$. Furuse, Influence of magnetic field on the brownian motion of charged particle, J. Phys. Soc. Japan 28, 559 (1970).
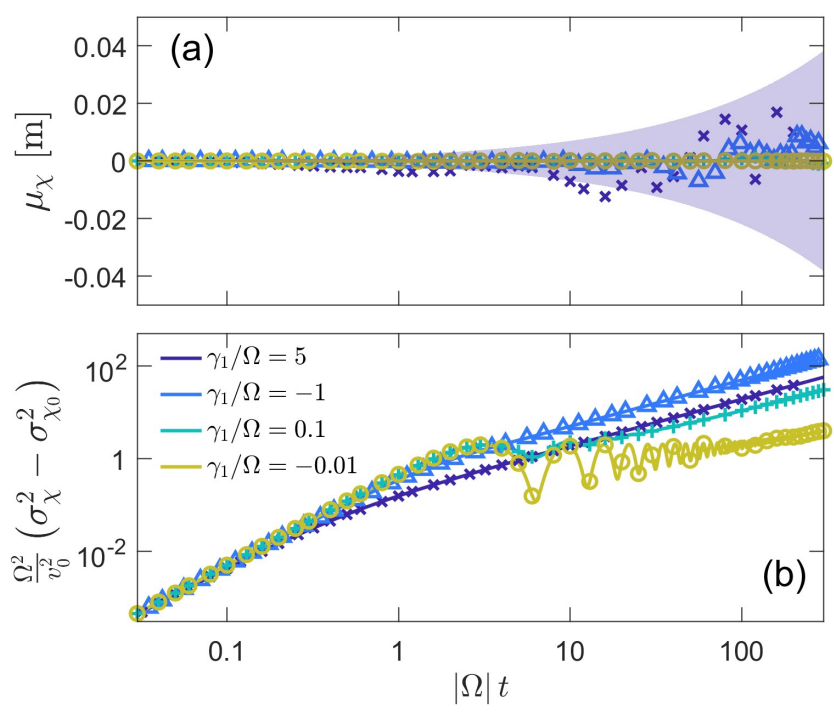

FIG. 6. Comparison between simulated (markers) and theoretical (solid lines) values of (a) $\mu_{\chi}(t)$ and (b) $\sigma_{\chi}^{2}(t)$ for different $\gamma_{1} / \Omega$ (colors as per legend). Different marker shapes represent different choices of simulation parameters as described in the text. The purple shading in (a) corresponds to the expected $1 \sigma$ statistical uncertainty area around the theoretical mean $\left(\mu_{\chi}(t)=0\right)$ for the simulations with $\gamma_{1} / \Omega=5$.

${ }^{7}$ R.F. Fox, Gaussian stochastic processes in physics, Phys. Rep. 48, 179-283 (1978).

${ }^{8}$ A. Bovet, Suprathermal ion transport in turbulent magnetized plasmas, Ph.D. dissertation, École Polytechnique Fédérale de Lausanne, Switzerland, 2015.

${ }^{9} \mathrm{~V}$. Lisy and J. Tothova, Brownian motion of charged particles driven by correlated noise in magnetic field, Trans. Theo. Stat. Phys. 42, 365 (2013).

${ }^{10}$ L.C. Evans, An introduction to stochastic differential equations, American Mathematical Society, 2013, pp. 86-89.

${ }^{11}$ I. Calvo, R. Sánchez and B.A. Carreras, Fractional Lévy motion through path integrals, J. Phys. A 42, 055003 (2009).

${ }^{12}$ A. Fasoli, I. Furno and P. Ricci, The role of basic plasmas studies in the quest for fusion power, Nature Phys. 15, 872-875 (2019).

${ }^{13}$ I. Furno, F. Avino, A. Bovet, A. Diallo, A. Fasoli, K. Gustafson, D. Iraji, B. Labit, J. Loizu, Müller, G. Plyushchev, M. Podesta, F.M. Poli, P. Ricci and C. Theiler, Plasma turbulence, suprathermal ion dynamics and code validation on the basic plasma physics device TORPEX, J. Plasma Phys. 81, 345810301 (2015).

${ }^{14}$ A. Bovet, M. Gamarino, I. Furno, P. Ricci, A. Fasoli, K. Gustafson, D.E. Newman and R. Sánchez, Transport equation describing fractional Lévy motion of suprathermal ions in TORPEX, Nucl. Fusion 54, 104009 (2014).

${ }^{15}$ A. Bovet, A. Fasoli, P. Ricci, I. Furno and K. Gustafson, Nondiffusive transport regimes for suprathermal ions in turbulent plasmas, Phys. Rev. E 91, 041101(R) (2015).

${ }^{16}$ F. Manke, M. Baquero-Ruiz, I. Furno, O. Chellaï, A. Fasoli and P. Ricci, Truncated Lévy motion through path integrals and applications to nondiffusive suprathermal ion transport, Phys. Rev. E 100, 052122 (2019).

${ }^{17}$ J. Masoliver, K. Lindenberg and G.H. Weiss, A continuous-time generalization of the persistent random walk, Physica A 157, 891 (1989).

18 J. Masoliver, J.M. Porrà and G.H. Weiss, Some two and threedimensional persistent random walks, Physica A 193, 469 (1993).

${ }^{19}$ M. Baquero-Ruiz, F. Manke, I. Furno, A. Fasoli and P. 
Ricci, Particle transport at arbitrary timescales with Poissondistributed collisions, Phys. Rev. E 100, 052134 (2019).

${ }^{20}$ H.G. Othmer, S.R. Dunbar and W. Alt, Models of dispersal in biological systems, J. Math. Biol. 26, 263 (1988).

${ }^{21}$ A. Gut, Probability: A graduate course, 2nd ed. Springer, New York, 2013.

${ }^{22}$ G.B. Arfken, H.J. Weber and F.E. Harris, Mathematical methods for physicists, 7th ed. Academic Press, Waltham MA, 2013.

${ }^{23}$ C. Forbes, M. Evans, N. Hastings and B. Peacock, Statistical distributions, 4th ed. John Wiley and Sons, New Jersey, 2011.

${ }^{24}$ B. Labit, A. Diallo, A. Fasoli, I. Furno, D. Iraji, S.H. Müller, G. Plyushchev, M. Podestà, F.M. Poli, P. Ricci, C. Theiler and J. Horaček, Statistical properties of electrostatic turbulence in toroidal magnetized plasmas, Plasma Phys. Control. Fusion 49, B281 (2007).

${ }^{25}$ M. Baquero-Ruiz, F. Avino, O. Chellai, A. Fasoli, I. Furno, R. Jacquier, F. Manke, and S. Patrick, Dual Langmuir-probe ar- ray for $3 D$ plasma studies in TORPEX, Rev. Sci. Instrum. 87 , 113504 (2016).

${ }^{26}$ C. Theiler, A. Diallo, A. Fasoli, I. Furno, B. Labit, M. Podestà, F.M. Poli and P. Ricci, The role of the density gradient on intermittent cross-field transport events in a simple magnetized toroidal plasma, Phys. Plasmas 15, 042303 (2008).

${ }^{27}$ P. Ricci, F.D. Halpern, S. Jolliet, J. Loizu, A. Mosetto, A. Fasoli, I. Furno and C. Theiler, Simulation of plasma turbulence in scrape-off layer conditions: the GBS code, simulation results and code validation, Plasma Phys. Control. Fusion 54, 124047 (2012).

${ }^{28}$ K. Gustafson, P. Ricci, A. Bovet, I. Furno, and A. Fasoli, Suprathermal ion transport in simple magnetized torus configurations Phys. Plasmas 19, 062306 (2012).

${ }^{29}$ B. Gutiérrez-Medina, A. Jiménez, A.I. Peña, Y. Covarrubias and J.V. García, Circular random motion in diatom gliding under isotropic conditions, Phys. Biol. 11, 066006 (2014). 